\title{
Autologous cell therapies: the importance of regulatory oversight
}

\author{
Michael Werner, Tim Mayleben \& Gil Van Bokkelen*
}

Promising new medical technologies and approaches to treatment offer the potential to substantially improve the lives of patients who need help, and in some cases transform the way medicine is practiced. In recent years, the biotechnology industry has generated many examples of new approaches that are having a profound impact on medical care, including protein and peptide therapeutics, monoclonal antibodies and molecular diagnostics.

\section{Few would argue that the regulatory landscape doesn't need improving, and we must continuously be thinking about ways to make it clearer, more predictable and more efficient, including refining the definitions used to determine regulatory oversight. ?}

Many regard the field of cell therapy in regenerative medicine as having transformational potential - capable of substantially improving medical outcomes, enhancing patient (and family) quality-of-life and ultimately reducing overall healthcare costs. Over the past decade, we have seen an explosion in the number of peerreviewed publications exploring the biology of various stem cell populations, and evaluating their use in a range of preclinical disease models. Furthermore, we have seen consistent growth in the number of programs advancing into clinical trials, using both autologous and allogeneic-based cell therapy approaches (TABLE 1).

Despite this, some argue that progress has been slowed by an overly burdensome regulatory framework imposed by the US FDA. While some FDA reforms are needed it is worth remembering that the majority of experimental therapies are ultimately found wanting during clinical trials. In some cases, promising candidate therapies do not actually help

Michael Werner, Athersys, Inc., (NASDAQ: ATHX), 3201 Carnegie Avenue, Cleveland 44115-2653, OH, USA

Tim Mayleben, Athersys, Inc., (NASDAO: ATHX), 3201 Carnegie Avenue, Cleveland 44115-2653, OH, USA

"Author for correspondence: Gil Van Bokkelen, Chairman \& CEO, Athersys, Inc., (NASDAQ: ATHX), 3201 Carnegie Avenue, Cleveland 44115-2653, OH, USA patients in a consistent or meaningful manner, or may pose unexpected safety issues that are only identified during the conduct of rigorous clinical studies. Even substances that naturally exist in the human body are not guaranteed to be safe and effective when they are used as therapies. In fact, while biologics have shown a meaningfully better clinical success rate than pharmaceuticals, only a minority of new biologic treatments are ultimately proven to be both safe and effective - approximately one in four, according to the BioMedtracker study results that were released in February 2010. This project, which analyzed over 4275 drug development programs in various stages of clinical development from October 2003 to December 2010, found that approval rates for lead indications among biologics was 26 versus $14 \%$ for other new molecular entities (and substantially lower for secondary indications in both categories).

Some have argued that a restrictive regulatory landscape in the USA has led many groups to conduct clinical trials internationally, foregoing trials that involve FDA oversight, thereby leading to a severe reduction of domestic clinical activity. However, an analysis of both autologous and allogeneic cell therapy-based approaches demonstrates 
that since 2000 , there are a substantial number of FDA-authorized clinical trials using stem cells that involve clinical institutions in the USA, and that the number of such trials has grown over time. While international clinical activity regarding stem cell therapies has also grown substantially, there clearly is no barrier to entry for sponsors who wish to conduct clinical studies in the USA (or in Europe, where the regulatory framework is generally regarded as being similar to that in the USA) (FIGURE 1).

The Center for Biologics Evaluation and Research is the center within FDA that regulates biological products for human use under applicable federal laws, including the Public Health Service Act and the Federal Food, Drug and Cosmetic Act. FDA regulations for cell therapies are designed to promote safe collection, manufacture, storage and use of human cells, and cellular- and tissuebased products (HCT/P). These regulations can be found at $21 \mathrm{CFR}$. Parts 1270 and 1271 (note that The Center for Biologics Evaluation and Research does not regulate the transplantation of vascularized human organ transplants, such as kidney, liver, heart, lung or pancreas). FDA regulations of $\mathrm{HCT} / \mathrm{Ps}$ include comprehensive requirements to prevent the introduction, transmission and spread of communicable disease.

To be exempt from FDA regulatory oversight HCT/Ps must meet criteria set forth in the Public Health Service Act. FDA defines 'manufacture' as "any or all steps in the recovery, processing, storage, labeling, packaging or distribution of any human cell or tissue, and the screening or testing of the cell or tissue donor", as described in 21 CFR. 1271(e). To be exempt the HCT/P must be: minimally manipulated; intended for homologous use as reflected by the labeling, advertising or other indications of the manufacturer's objective intent; not be combined with a drug or device;
Table 1. Number of clinical trials listed on ClinTrials.gov analyzed by trial start date according to the year of indicated initiation.

\begin{tabular}{|lll|}
\hline Year & Autologous trials initiated & Allogeneic trials initiated \\
\hline $2012^{\dagger}$ & 22 & 22 \\
\hline 2011 & 50 & 23 \\
\hline 2010 & 47 & 12 \\
\hline 2009 & 45 & 15 \\
\hline 2008 & 30 & 18 \\
\hline 2007 & 34 & 10 \\
\hline 2006 & 29 & 10 \\
\hline 2005 & 32 & 6 \\
\hline 2004 & 23 & 5 \\
\hline 2003 & 12 & 14 \\
\hline 2002 & 13 & 9 \\
\hline 2001 & 12 & 17 \\
\hline $\begin{array}{l}\text { NNumbers for 2012 indicate trials initiated through mid-year. } \\
\text { Data from [1]. }\end{array}$ & \\
\hline
\end{tabular}

not have a systemic effect and not be dependent on the metabolic activity of living cells for its primary function except if for autologous use, allogeneic use in a first-degree or second-degree blood relative, or reproductive use. Additional components of the FDA's regulation of HCT/Ps are found at 21 CFR 1271.

The concept of what constitutes appropriate regulatory oversight was the central issue in a recent ruling by the United States District Court for the District of Columbia in United States $v$. Regenerative Sciences LLC. This case raised important questions about the extent of the FDA's jurisdiction over procedures where autologous cells (a patient's own cells or tissues) are administered in a physician's office, clinic or a hospital. At the heart of the argument is whether such treatments are 'biologic medicines' (also known as a 'biologic' or a 'biological product'), that should require human clinical trials to demonstrate safety and efficacy and be regulated by FDA.

In autologous cell therapy, where cells are isolated from the patient for subsequent readministration to the patient, the FDA has stipulated that in some circumstances, no clinical trials are required. Specifically, this is the case if the cells or tissue that have been harvested from the patient meet the standard of 'minimally manipulated' and the cells are being used in a 'homologous manner'. For autologous therapies that do not meet the definition of minimally manipulated, and/or that are not being used for a homologous biological purpose, the FDA has deemed it appropriate to require clinical trials to establish safety and efficacy.

Key to understanding whether FDA regulations apply are the concepts 'homologous use'. The FDA defines 'minimal manipulation' in the following manner: “...[f]or cells or nonstructural tissues, processing that does not alter the relevant biological characteristics of cells or tissues" (see 21 CFR 1271.3(f)(2)). While the FDA has issued some guidance on the minimal manipulation of structural tissue, no such guidance has been issued to define the minimal manipulation of cells. However, in a proposed approach issued in 1997, the Agency stated that "processing of cells and nonstructural tissues to be more-than-minimal manipulation when the processing of 'minimally manipulated' and 
alters the biological characteristics (and thus potentially the function or integrity) of the cells or tissue, or when adequate information does not exist to determine whether the processing will alter the biological characteristics of the cell or tissue. Examples of morethan-minimal manipulation of cells and tissues include cell expansion, encapsulation, activation, or genetic modification" [Docket Number 97N-0068]. FDA regulations define 'homologous use' as "the repair, reconstruction, replacement, or supplementation of a recipient's cells or tissues with an HCT/P that performs the same basic function or functions in the recipient as in the donor" (see 21 C.F.R. 1271.3(c)). The transplantation of hematopoietic stem cells obtained from bone marrow or the peripheral blood for the treatment of leukemia is an example of homologous use, and is a treatment that has been used and studied for more than 40 years.
Some argue that this regulatory treatment of autologous cell therapies may be contrary to good medical practice. They say physicians must be allowed to use their judgment to develop and administer new cell therapies outside of the clinical trial process - similar to other experimental surgical or medical procedures. Clearly, there needs to be some room for investigator-driven innovation in medical practice, and physician-sponsored clinical studies. However, it is also important to ensure that patients are being adequately informed with regard to the true risks and benefits of a potential treatment especially when they are asked to pay for it. Unfortunately, while in general there is an outstanding record of safety associated with autologous cell therapies, there are a few examples where a patient's own cells have been readministered for something other than a homologous biological purpose, in which the patient was inadvertently harmed, or even died, as a result. These types of events could reflect a lack of training or knowledge that could be gained from formal clinical trials, and underscore the need for some form of rigorously controlled studies and regulatory oversight. It should not be assumed that just because the cells are derived from the patient that the approach is perfectly safe, the physician and patient are both adequately informed, and nothing bad can happen.

Few would argue that the regulatory landscape doesn't need improving, and we must continuously be thinking about ways to make it clearer, more predictable and more efficient, including refining the definitions used to determine regulatory oversight. Recent progress should help create a clearer and more efficient regulatory path for sponsors attempting to develop new therapies designed to address significant unmet medical needs. In July, Congress passed and President Obama signed the Federal Drug and

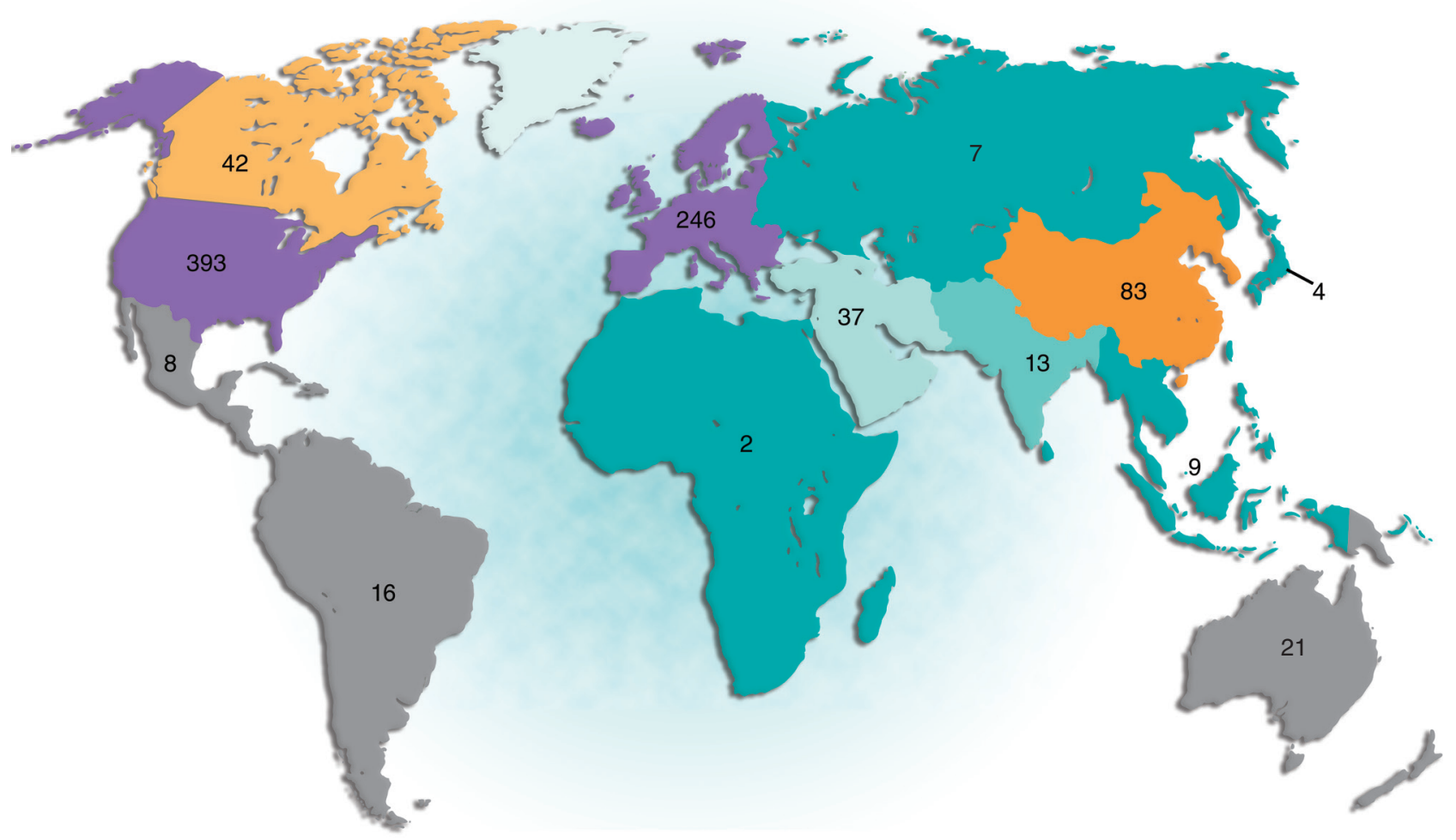

Figure 1. Geographic distribution of clinical trials that have been registered on ClinTrials.gov since January 2000 that are listed as involving stem cells as an interventional part of the therapy. Search criteria exclude gene therapy trials. 
Safety Innovation Act, which included a renewal of the Prescription Drug User Fee Act. This legislation included important new provisions intended to speed clinical development in areas of great medical need. Specifically, it includes a broadening of the 'accelerated approval' pathway, and the creation of a 'breakthrough therapies' category for promising new medicines. Both frameworks are designed to reduce the length, cost and complexity of clinical trials, while also protecting patient safety. Taken together, these provisions are likely to greatly facilitate rapid development and commercialization of regenerative medicine and cell therapies, so that patients can be helped.

Current regulations balance protecting public health by requiring data demonstrating that certain uses of cells are safe and effective. How they are applied will likely change over time as science advances and researchers and the FDA - learn more about the biological properties of certain cell and tissue based therapies. The regulatory and clinical trial process is intended to ensure public safety during this time of scientific advancement.
But companies that promote cell and tissue 'therapies' not demonstrated to be safe and effective are really only marketing risk, veneered with unsubstantiated promises. When adverse events happen under those conditions, all patients are hurt because it slows the field, delaying the development of safe and effective cures and treatments. Thoughtful, rigorous science will ultimately bring safe and effective products to patients.

Physicians must always be empowered to do whatever they lawfully can and use their best medical judgment to help patients. But ultimately, patients need their physicians to have access to numerous safe and effective therapies to treat their disease or condition. The best - and fastest - way to achieve that goal is to ensure that cell therapies undergo rigorous testing.

The prospect of new regenerative treatments and even cures where current medicine and therapies offer little hope for the patient is thrilling. The very promise of regenerative medicine illustrates the need for regulation: for where there is promise science provides protection against false hope. A rigorous clinical trial system will ensure that safe and effective products, especially those with transformational potential, reach patients quickly.

\section{Financial \& competing interests}

disclosure

G Van Bokkelen is employed as Chairman and CEO by, owns shares in and has options with Athersys. T Mayleben is CEO at and holds financial interest in Aastrom. M Werner is a partner at Holland \& Knight, a law firm that represents numerous companies in the regenerative medicine sector. The authors have no other relevant affiliations or financial involvement with any organization or entity with a financial interest in or financial conflict with the subject matter or materials discussed in the manuscript apart from those disclosed.

No writing assistance was utilized in the production of this manuscript.

\section{Website}

1 ClinicalTrials.gov http://clinicaltrials.gov 